71 A GENOME-WIDE ASSOCIATION STUDY IN INDIAN ASIANS IDENTIFIES FOUR SUSCEPTIBILITY LOCI FOR TYPE-2 DIABETES

doi:10.1136/heartjnl-2011-300198.71

\begin{abstract}
${ }^{1,2} \mathrm{~J}$ Sehmi, ${ }^{3} \mathrm{D}$ Salaheen, ${ }^{4} \mathrm{Y}$ Yeo, ${ }^{5} \mathrm{~W}$ Zhang, ${ }^{1,2} \mathrm{D}$ Das, ${ }^{6} \mathrm{M}$ I McCarthy, ${ }^{4} \mathrm{E} S$ Tai, ${ }^{3} \mathrm{~J}$ Danesh, ${ }^{1,2} \mathrm{~J}$ Kooner, ${ }^{2,7} \mathrm{~J}$ Chambers. ${ }^{1}$ National Heart and Lung Institute, Imperial College, London, UK; ${ }^{2}$ Ealing Hospital NHS Trust, London, UK; ${ }^{3}$ Department of Public Health and Primary care, Cambridge University, Cambridge, UK; ${ }^{4}$ Department of Medicine, National University of Singapore, Singapore; ${ }^{5}$ Department of Epidemiology and biostatistics, Imperial College London, London, UK; ${ }^{6}$ Wellcome Trust for human Genetics, Oxford University, Oxford, UK; ${ }^{7}$ Department of Epidemiology and biostatistics, Imperial College London, London, UK
\end{abstract}

Background Type-2 diabetes (T2D) is a major risk factor for cardiovascular disease, and a leading causing of mortality worldwide. T2D is $2-4$ fold more common among Indian Asians than Europeans, and contributes to higher cardiovascular disease mortality in Asians. Little is known of the genetic basis of T2D in Indian Asians. Methods We carried out a genome-wide association (GWA) study of T2D in 5561 Indian Asian cases and 14458 controls from LOLIPOP, PROMIS and SINDI cohorts. Whole genome scans were performed using the Illumina $317 \mathrm{k}$ or $610 \mathrm{k}$ arrays. Further testing of suggestive SNPs was carried out in independent cohorts of Indian Asian $(12 \mathrm{~K} \mathrm{~T} 2 \mathrm{D}$ cases and $25 \mathrm{~K}$ controls) and European ancestry (DIAGRAM+, $8 \mathrm{~K} \mathrm{T2D}$ cases and $39 \mathrm{~K}$ controls).

Results There were two novel loci associated with T2D at $\mathrm{p}<10^{-6}$, and an additional 57 loci associated with T2D at $\mathrm{p}<10^{-4}$ in the GWA study. We used results from DIAGRAM+ to prioritise 19 loci for further testing in Indian Asians. In combined analysis of results from GWA and further testing, four loci now reached genome-wide significance $\left(p<5 \times 10^{-8}\right)$ among Indian Asians. Coding variant and eQTL studies at these loci identify genes closely involved in insulin secretion and signalling.

Conclusion We identify four novel genetic loci associated with T2D in Indian Asians. Our observations provide new insights into the biological mechanisms underlying $\mathrm{T} 2 \mathrm{D}$, a major risk factor for cardiovascular disease.

\section{ANGIOGENESIS IN RESPONSE TO UPREGULATED HYPOXIC SIGNALLING IS DEPENDENT ON HAEMODYNAMIC FLOW}

doi:10.1136/heartjnl-2011-300198.72

${ }^{1} \mathrm{O}$ J Watson, ${ }^{1} \mathrm{~F} \mathrm{~J}$ van Eeden, ${ }^{1} \mathrm{C}$ Gray, ${ }^{2} \mathrm{~T}$ J A Chico. ${ }^{1} \mathrm{M} R \mathrm{C}$ Centre for Developmental Biology and Genetics, University of Sheffield, Sheffield, UK; ${ }^{2}$ NIHR Cardiovascular Biomedical Research Unit, Sheffield Teaching Hospitals NHS Foundation Trust, Sheffield, UK

Introduction Hypoxia drives angiogenesis in a range of pathologies. Mutations in von hippel lindau protein (vhl) lead to excessive angiogenesis via upregulation of hypoxic signalling, due to impaired HIF-1 $\alpha$ degradation. Physical forces exerted by blood flow have been shown to contribute to vascular remodelling. We therefore used vhl mutant zebrafish to observe the interplay between hypoxic signalling, haemodynamic flow and vascular development. Since NO has been shown to be both pro-angiogenic and released in response to haemodynamic force, we assessed whether NO contributed to angiogenesis in this model.

Methods Vhl mutant zebrafish were crossed with a fli1; GFP transgenic that expresses Green Fluorescent Protein (GFP) in the endothelium. Embryonic vascular development was observed in mutants and wild type siblings by confocal microscopy. To determine the role of blood flow in the angiogenic response, cardiac troponin $t_{2}$ was knocked down by morpholino antisense injection. To assess the contribution of nitric oxide, embryos were treated with either L-NAME (nitric oxide synthase inhibitor) $(1 \mathrm{mM})$ or sodium nitroprusside ( $\mathrm{NO}$ donor) $(100 \mu \mathrm{M})$ from $24-\mathrm{h}$ post fertilisation (hpf) until imaging at $4 \mathrm{dpf}$.

Results Imaging of the developing trunk vasculature revealed that vhl mutant embryos display excessive and aberrant angiogenesis from 3dpf (Abstract 72 figure 1A, B). Cardiac troponin $\mathrm{T}_{2}$ knockdown prevented any cardiac contraction, but embryos develop normally due to passive oxygen diffusion. Loss of blood flow did not alter normal intersegmental vessel patterning in either controls (Abstract 72 figure 1C) or vhl mutants (Abstract 72 figure 1D) However, loss of blood flow completely prevented excessive angiogenesis in vhl mutants (Abstract 72 figures $1 \mathrm{D}$ and 2), implying that both blood flow and hypoxic signalling are required for "pathological" angiogenesis but not developmental angiogenesis (vasculogenesis). NO synthase inhibition with L-NAME had no effect, suggesting that the contribution of flow to excessive angiogenesis in response to upregulated hypoxic signalling is $\mathrm{NO}$ independent.
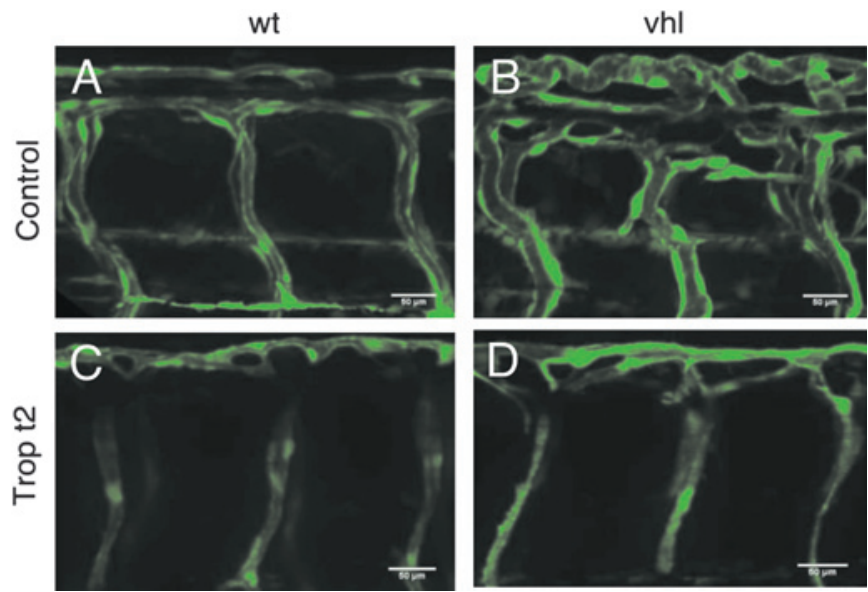

Abstract 72 Figure 1

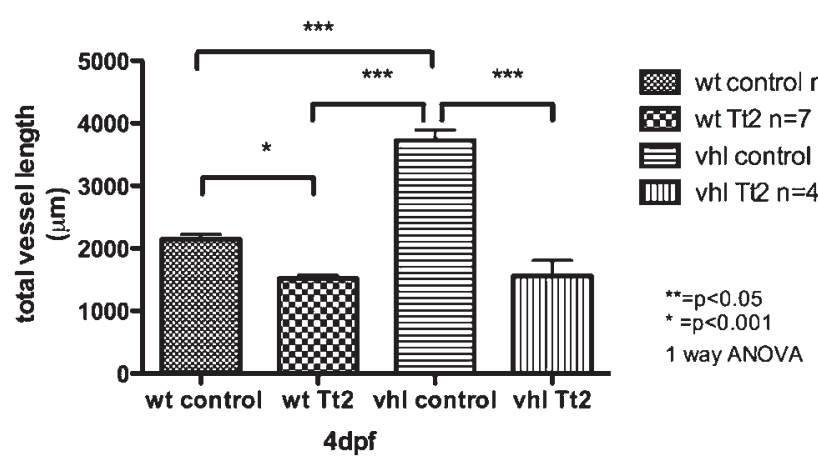

Abstract 72 Figure 2 Effect of troponin T knockdown on total vessel length.

Conclusion Angiogenesis in response to hypoxic signalling is critically dependent upon haemodynamic force, compared with developmental vasculogenesis that can proceed in the absence of any blood flow. This indicates a different mechanism of development for hypoxia driven angiogenesis and vasculogenesis which may have important therapeutic implications.

\section{HERITABILITY OF CORONARY FLOW RESERVE}

doi:10.1136/heartjnl-2011-300198.73

R Ahmed, P Muckett, S Cook. Clinical Sciences Centre, Imperial College, London, UK

Introduction Coronary flow reserve (CRF) is the ratio of peak coronary flow during maximal coronary artery dilatation to basal 